\title{
Uso de dispositivos móviles para el desarrollo de las prácticas de laboratorio utilizando una herramienta on line
}

\author{
Santiago Lillo ${ }^{a}$, María del Mar Camachob y Nuria Martínez-Navarrete ${ }^{\mathrm{c}}$ \\ ${ }^{a}$ Departamento de Expresión Gráfica Arquitectónica (Universitat Politècnica de València, \\ sanlilgi@ega.upv.es), bepartamento de Tecnología de Alimentos (Universitat Politècnica de \\ València, mdmcamvi@tal.upv.es) y ${ }^{c}$ Departamento de Tecnología de Alimentos (Universitat \\ Politècnica de València,nmartin@tal.upv.es)
}

\begin{abstract}
Laboratory practices allow students to learn concepts and develop competencies that contribute to making sense of the theory of the subject in question. That is why, in many cases, they play a primary role in the overall teaching load of the subject, so that their assessment may become very important in the final grade. Thus, its monitoring and evaluation acquire significant relevance. In this sense, it would be of great interest to implement the use of a tool that would guide the student in the development of the work and help him to systematically collect the relevant data for further treatment, in addition to facilitating the final evaluation by the teacher. It would also be desirable that, at the same time, the tool facilitate the final evaluation by the teacher. Based on these premises, the use of mobile devices as a pedagogical tool is proposed, since its infinite applications allow to complement the learning process taking into account some of its advantages. One of them may be the increased motivation of the students when incorporating to their academic tasks a tool with which they are fully familiar. The objective of the present work is to implement a new methodology that guides the student in the realization of the practices through the incorporation of mobile devices. Its use in laboratory practices allowed to provide order to the data obtained from the development of the work and greater accessibility to information by all students. At the same time, it allowed the teacher a simple control of the attendance to the practice and facilitated the evaluation of the practical work of the student.
\end{abstract}

Keywords: Mobile devices, surveys, evaluation, laboratory practices.

\begin{abstract}
Resumen
Las prácticas de laboratorio permiten a los estudiantes aprender conceptos y desarrollar competencias que contribuyen a dar sentido a la teoría del tema en cuestión. Es por esto que, en muchos casos, tienen un peso determinante en la carga docente global de la asignatura, de forma que su valoración puede llegar a ser muy importante en la nota final. Así, su seguimiento y evaluación adquieren una relevancia significativa. En este sentido, sería de gran interés implementar el uso de alguna herramienta que permitiese guiar
\end{abstract}


al alumno en el desarrollo del trabajo y le ayudase a recoger sistemáticamente los datos pertinentes para su posterior tratamiento, además de facilitar la evaluación final por parte del profesor. En base a estas premisas, se propone el uso de los dispositivos móviles como herramienta pedagógica, puesto que sus infinitas aplicaciones permiten complementar el proceso de aprendizaje aprovechando algunas de sus ventajas. Una de ellas puede ser el aumento de la motivación por parte de los alumnos, al incorporar a sus tareas académicas una herramienta con la que se encuentran plenamente familiarizados. El objetivo del presente trabajo es implementar una nueva metodología que guíe al alumno en la realización de las prácticas a través de la incorporación de los dispositivos móviles. Su uso en las prácticas de laboratorio permitió proporcionar orden a los datos obtenidos del desarrollo del trabajo y una mayor accesibilidad a la información por parte de todos los alumnos. Al mismo tiempo, al profesor le permitió un sencillo control de la asistencia a la práctica y le facilitó la evaluación del trabajo práctico del alumno.

Palabras clave: Dispositivos móviles, encuestas, evaluación, prácticas de laboratorio.

\section{Introducción}

El rol de los profesores como facilitadores del aprendizaje debe llevarnos al uso de una variedad de recursos. Bain (2007) presenta una interesante investigación sobre profesores excelentes y muestra que muchos de estos profesores ayudan a sus alumnos con muy buenas clases magistrales, pero otros se apoyan más en otras estrategias didácticas y en otros recursos. Se pueden diseñar tareas de aprendizaje muy variadas (para clase y para fuera de clase) que literalmente responden a un enfoque didáctico centrado en lo que hace el alumno y que constituyen a su vez un eficaz sistema de evaluación (Morales Vallejo, 2008). Hay que destacar la necesidad de fomentar la pluralidad metodológica para atender la diversidad de personas, situaciones y ámbitos de conocimiento que existen. Entre las estrategias didácticas pueden utilizarse métodos de transmisión (lección magistral), métodos de enseñanza comprensiva no lineal (clases de resolución de problemas, prácticas en aula informática, prácticas de laboratorio, entre otros) y métodos de enseñanza no formal (los que surgen en contextos de intercambio, foros, seminarios abiertos, etc). De todos ellos, tradicionalmente la lección magistral ha sido el foco de atención desde el punto de vista de acciones de mejora. Sin embargo, las prácticas de laboratorio constituyen una parte imprescindible de la estructura de muchas asignaturas, ya que intervienen en la formación de los alumnos complementando la parte teórica. En particular permiten a los estudiantes aprender conceptos y desarrollar competencias en la aplicación de conocimientos, habilidades y actitudes en situaciones reales. De esta forma dan sentido a la teoría, al llevarla a la práctica, y aprenden a reconocer los aspectos más importantes y los problemas inherentes a ella. Una de las herramientas más utilizadas para la evaluación del aprendizaje en las prácticas de laboratorio es el informe, que se suele presentar días después de la 
realización de la práctica y que acaba siendo muy laborioso, en algunos casos, y un mero plagio entre alumnos, en otros. En este sentido, alguna herramienta que permita la recogida sistemática de datos durante la ejecución de las prácticas, para su posterior tratamiento en relación con los conceptos adquiridos en las sesiones de teoría, sería de gran interés. En la bibliografía se han descrito tareas que permiten este tipo de actividades, como la presentación de cuestionarios o tablas que deben completarse (Noguera, Tortajada, Atienza \& Herrero, 2011) y que pueden ayudar al profesor a la evaluación global de las prácticas, pero que, por otra parte, podrían ser susceptibles de mejora si se combinan con la utilización de nuevas tecnologías (Muñoz et al., 2016).

A pesar de la plena integración en la sociedad actual de los dispositivos móviles y, en mayor medida si cabe, en el colectivo universitario, su uso como herramienta pedagógica no se encuentra en la actualidad tan extendido en virtud de las posibilidades que puede ofrecer, a pesar de que existen, sin embargo, algunos precedentes tales como el m-learning (mobile learning). Se trata de una metodología que complementa el proceso de aprendizaje, a través de instrumentos móviles, computadoras portátiles, tablets, lectores de MP3, teléfonos inteligentes (smartphones) y teléfonos móviles y tiene infinitas aplicaciones, dependiendo de la creatividad del docente (Gisbert \& Esteve, 2011). Éstos pueden incidir de forma negativa en la atención de los alumnos durante las clases, sin embargo, de su utilización como herramienta pedagógica en el ámbito académico se derivan algunas ventajas a nivel global. Una de las más inmediatas es el aumento, por parte de los alumnos, de la motivación y de la predisposición al proceso de aprendizaje, al incorporar a sus tareas académicas una herramienta con la que se encuentran plenamente familiarizados y que, por tanto, pueden incorporar a su flujo de trabajo de una manera rápida (Andrés-Ferre, AlfaroCid, Lillo, Pérez de los Cobos \& Rodrigo, 2013).

\section{Objetivo}

El objetivo del presente trabajo es implementar una nueva metodología que guíe al alumno en la realización de las prácticas, a través de la incorporación de los dispositivos móviles. Esta metodología debe permitir generar bases de datos que serán utilizadas, a su vez, en fases posteriores de la práctica para su análisis y posible relación con los conocimientos teóricos adquiridos en la asignatura.

\section{Desarrollo de la innovación}

Como ejemplo para la aplicación de la propuesta de mejora se ha seleccionado una asignatura del Máster Universitario en Ciencia e Ingeniería de los Alimentos, Tecnología de los alimentos en polvo, impartido en la Escuela Técnica Superior de Ingeniería Agronómica y del Medio Natural en la Universitat Politècnica de València. Es una asignatura optativa de 2,5 créditos, con pocos alumnos ( $<25)$ y con una carga práctica importante $(1,25$ créditos), por lo que la valoración de las prácticas de laboratorio tiene un peso significativo en la nota final de la asignatura (40\%). Las sesiones de prácticas se organizan de manera que los 
alumnos trabajan cada uno con una muestra diferente, de forma que la última sesión se dedica a la puesta en común de todos los resultados.

Durante el curso pasado 2016-2017, para el seguimiento y evaluación de las prácticas de esta asignatura, se sustituyó el clásico informe por una serie de tablas y cuestionarios que los alumnos debían ir completando durante las sesiones prácticas hasta elaborar una especie de dossier con todos los datos recogidos y su tratamiento en relación con los conceptos teóricos adquiridos. La innovación en el curso 2017-2018 ha consistido en la utilización de encuestas a través de dispositivos móviles, como sustitutas de las tablas y cuestionarios. La aplicación utilizada para ello ha sido la herramienta gratuita de Google Formularios de Google. La selección de esta herramienta se hizo en base a su accesibilidad y facilidad de uso. Además, es compatible con todos los sistemas operativos móviles (IOS y Android) y con cualquier navegador de internet. Por otra parte, no es necesaria ninguna instalación previa, tan solo un dispositivo con acceso a internet.

Teniendo como base el material utilizado en el curso anterior, se han elaborado las preguntas que deberán incluirse en las encuestas que se responderán a través del móvil. Estas preguntas, que el alumno debe responder de forma individual, sirven además como una guía que facilita el proceso global de la práctica, estableciendo un orden lógico de las etapas que deberá ir completando hasta su resolución final. Una vez elaboradas las encuestas, se facilita a los alumnos la url previamente acortada, con el fin de que el proceso de introducción de la dirección electrónica en el móvil sea más rápido y con menos errores.

A pesar de que la toma de datos se lleva a cabo de forma grupal (en parejas o tríos) la encuesta debe ser respondida individualmente. Para ello, el primer campo que deben rellenar los alumnos es el nombre, de forma que sirva además como comprobante de la asistencia a las prácticas (Figura 1).

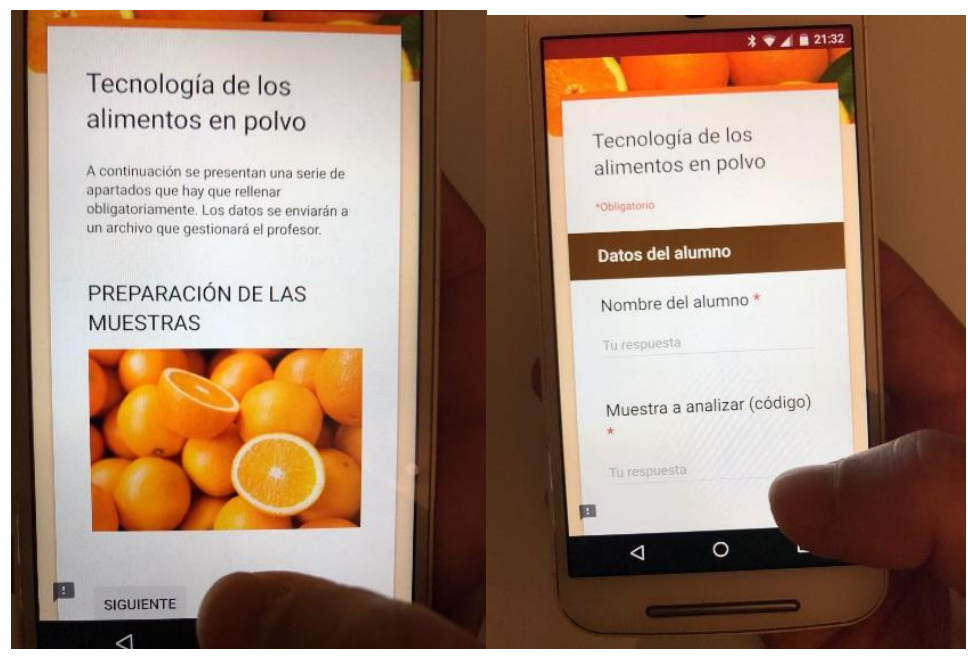

Fig. 1 Ejemplo del inicio de una de las encuestas y primer campo a rellenar por el alumno. Campo obligatorio. 
Se planteó en un principio añadir a cada pregunta la opción "respuesta obligatoria" que incluye el formulario Google, con el fin de garantizar que todas quedan respondidas, lo cual es fundamental para el correcto desarrollo de la práctica. Sin embargo, esta opción exige un desarrollo excesivamente lineal de la práctica, puesto que no ofrece la posibilidad de volver atrás y corregir las respuestas ya introducidas, en el caso de que se detecte algún error, lo cual suele ser bastante habitual. Para solventar este tipo de incidencias se eliminó la obligatoriedad de la respuesta y se incluyó una pregunta final del tipo "¿has rellenado todos lo campos de la encuesta?” . Esta pregunta, si bien no garantiza que no quede ningún campo sin completar, sí estimula al menos a que el alumno compruebe que, efectivamente, se ha respondido por completo a la encuesta (Figura 2). En este sentido, hemos echado en falta algún tipo de opción que impidiera enviar el resultado definitivo de la encuesta en el caso de que algún campo hubiera quedado sin completar, o bien, que todas las respuestas fueran de carácter obligatorio pero con la posibilidad de alterar el orden de su resolución.

Una vez terminada la sesión de prácticas, los alumnos han debido completar la encuesta correspondiente $\mathrm{y}$, automáticamente, se genera una tabla de resultados exportable como documento de hoja de cálculo. Esta hoja se pone a diposición delos alumnos a través de la plataforma propia de la UPV, poliformaT, para su tratamiento, en el caso de que sean datos, o para su comparación con los de los demás compañeros, en el caso de que ya sean resultados del tratamiento de dichos datos. Además, estas tablas generadas servirán para la evaluación de la parte práctica de la asignatura por parte del profesor.

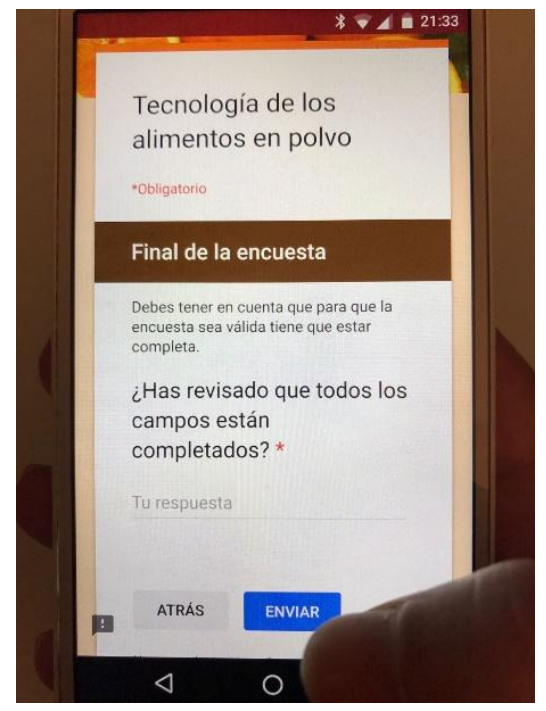

Fig. 2. Final de la encuesta. Campo obligatorio.

Por último y para conocer la opinión de los alumnos en cuanto a esta nueva metodología de aprendizaje, se realizó una encuesta de satisfacción en la que se preguntó sobre la contribución del móvil a organizar la toma de datos en las prácticas de laboratorio, su uso 
como guía o facilitando el aprendizaje de la materia. Por otra parte, también se les preguntó si su utilización había aumentado el interés en la asignatura y si pensaban que su uso era más eficaz que el uso del sistema “tradicional” (lápiz y papel). Por último, se les instó a sugerir mejoras en el sistema.

\section{Resultados}

A lo largo del curso, en las sesiones prácticas, se enviaron varios tipos de encuestas: de adquisición de datos, de cálculos y de razonamiento (Figura 3).

En la Figura 4 se presenta un momento de las prácticas en que los alumnos completan las encuestas in situ. El tipo de respuesta, numérica o corta, facilitó su cumplimentación. Así, las encuestas fueron enviadas al finalizar cada una de las sesiones prácticas.

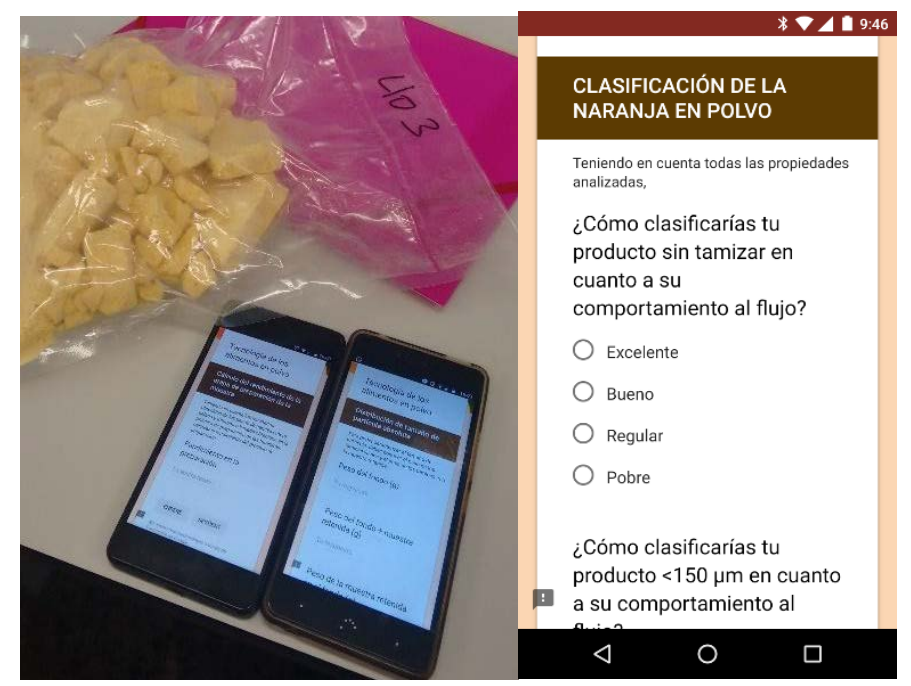

Fig. 3. Ejemplo de algunas de las cuestiones presentadas en las diferentes clases de encuestas: de adquisición de datos, de cálculos y de razonamiento. 


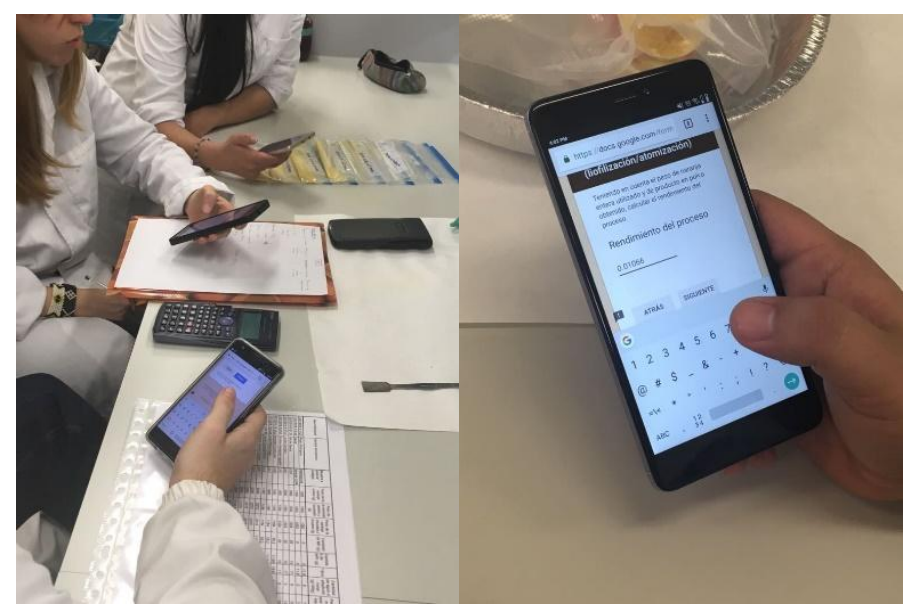

Fig. 4. Cumplimentación de las encuestas por parte del alumnado en el laboratorio.

En la Figura 5 se muestra, como ejemplo, una de las tablas de datos generada a partir de la introducción de éstos mediante el dispositivo móvil. Si se compara con la tabla de datos presentada en papel del curso 2016-17, después de haber sido revisa por el profesor (Figura 6), es evidente la diferencia. La presentación de la primera permite tener los datos más ordenados, haciendo más cómoda la tarea del profesor a la hora de comprobar que están bien para poderlos poner a disposición de los alumnos a través de la plataforma de la UPV poliformaT. Para los alumnos, la información está también más clara y ordenada. En las prácticas de esta asignatura, en concreto, cada grupo de alumnos trabaja con una muestra diferente que tiene que comparar con las de los demás compañeros, por lo que es también interesante tener todos los datos a disposición de todos en todo momento. Esto no ocurría cuando se utilizó la metodología del curso 16-17, ya que sólo estaba disponible una sola tabla para todos los alumnos que iba circulando de mano en mano hasta ser completada.

Encuesta 1 Preparación Muestra (respuestas).xlsx ty the

Archivo Editar Ver Insertar Formato Datos Herramientas Complementos Ayuda Todos los cambios se han guardado en Drive

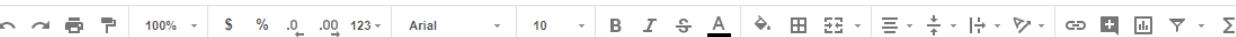

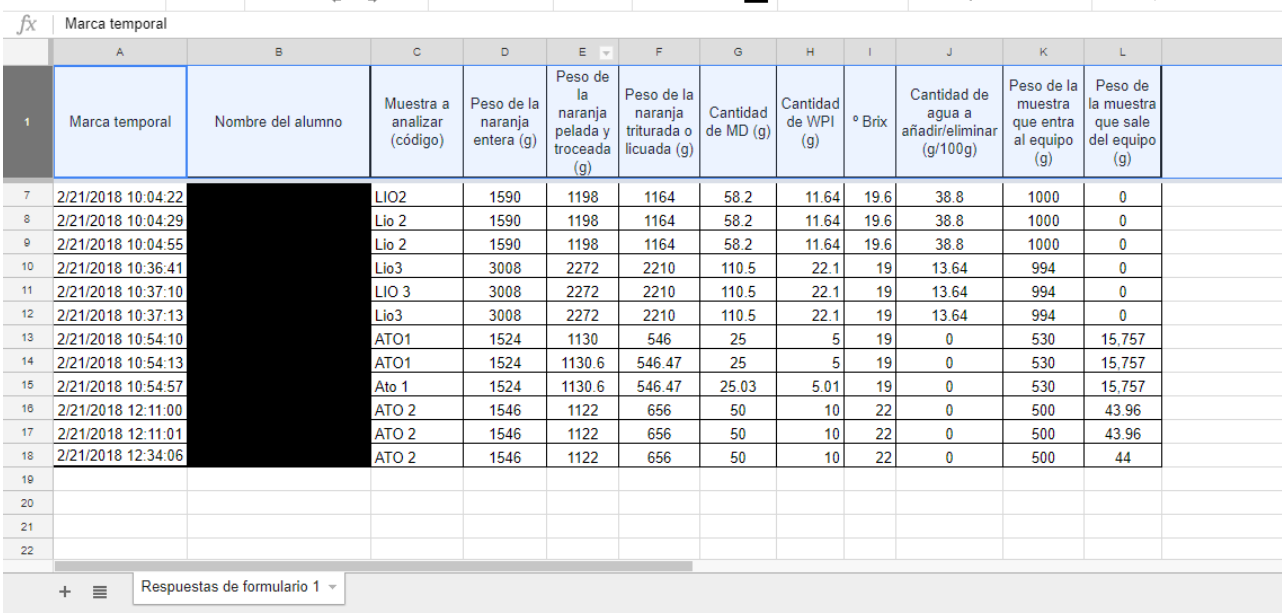

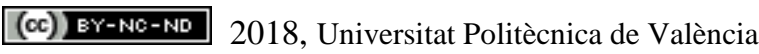

Congreso In-Red (2018) 
Fig. 5. Hoja de cálculo generada tras el envío de las encuestas cumplimentadas por los alumnos en las prácticas del curso 2017-18.

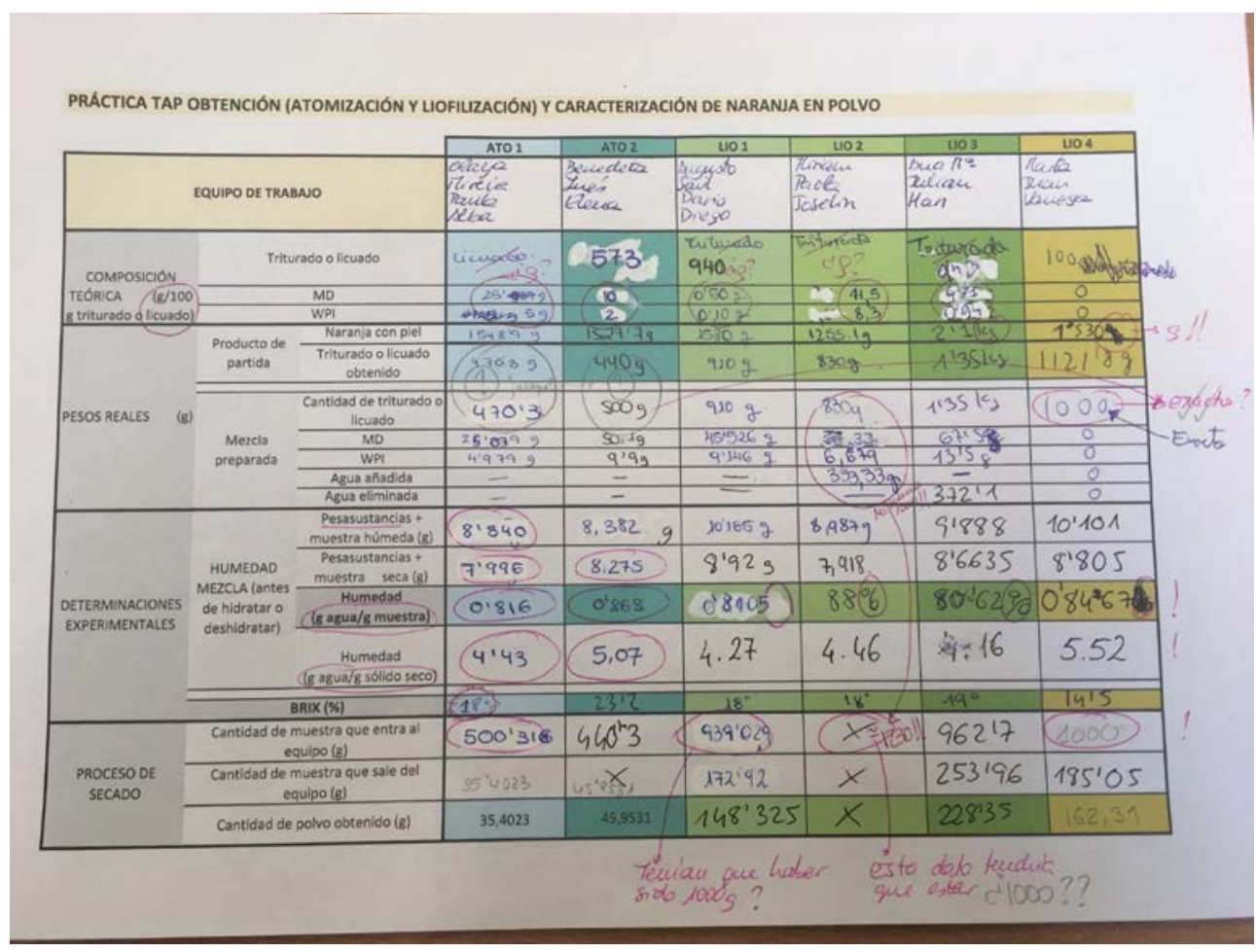

Fig. 6. Documento generado por los alumnos tras la toma de datos en las prácticas del curso 2016-17.

Estos datos son necesarios para la continuación de la práctica ya que a partir de ellos se tendrán que realizar cálculos que formarán parte de las respuestas de las encuestas siguientes. Las figuras 7 y 8 muestran los documentos generados en los cursos 2016-17 y 2017-18, respectivamente, con los resultados obtenidos a partir del tratamiento de los datos anteriormente recopilados.

(c)) EY-NC-ND 2018, Universitat Politècnica de València 


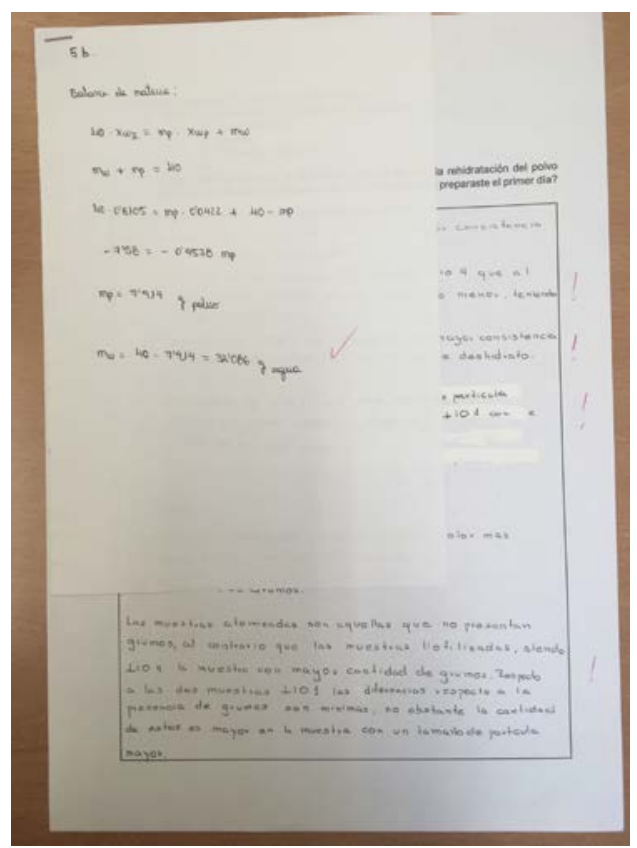

Fig. 7. Documento generado en el curso 2016-17 con los resultados obtenidos a partir del tratamiento de los datos anteriormente recopilados en el laboratorio.

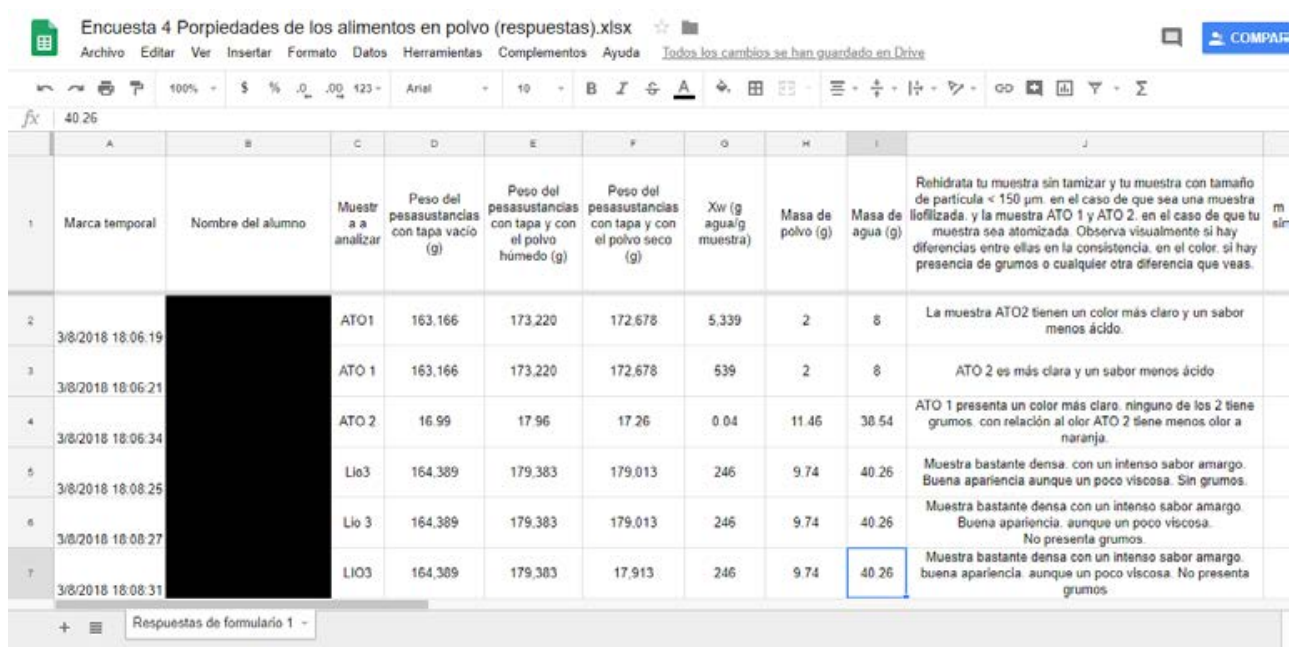

Fig. 8. Documento generado en el curso 2017-18 con los resultados obtenidos a partir del tratamiento de los datos anteriormente recopilados en el laboratorio.

En la última sesión práctica, la puesta en común de resultados, en la encuesta se presentan una serie de cuestiones para que el alumno analice los resultados obtenidos a lo largo de todas las prácticas y los relacione con los conceptos aprendidos en teoría. Las figuras 9 y 10 muestran un ejemplo de los documentos de los cursos 2016-17 y 2017-18, respectivamente.

(c)) EY-NC-ND 2018, Universitat Politècnica de València 


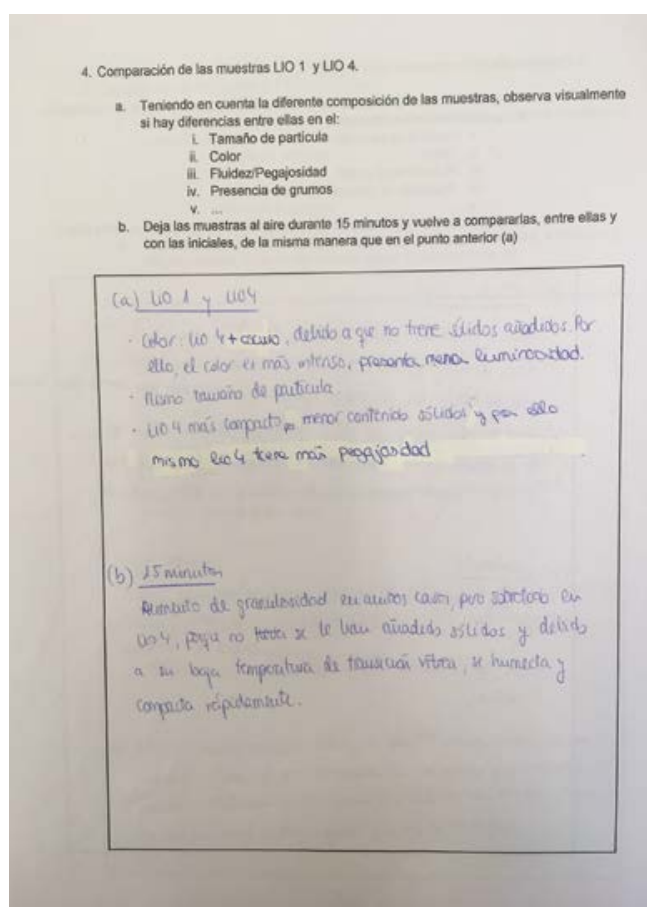

Fig. 9. Documento generado de la puesta en común de los resultados de las prácticas de laboratorio en el curso 2016-17.

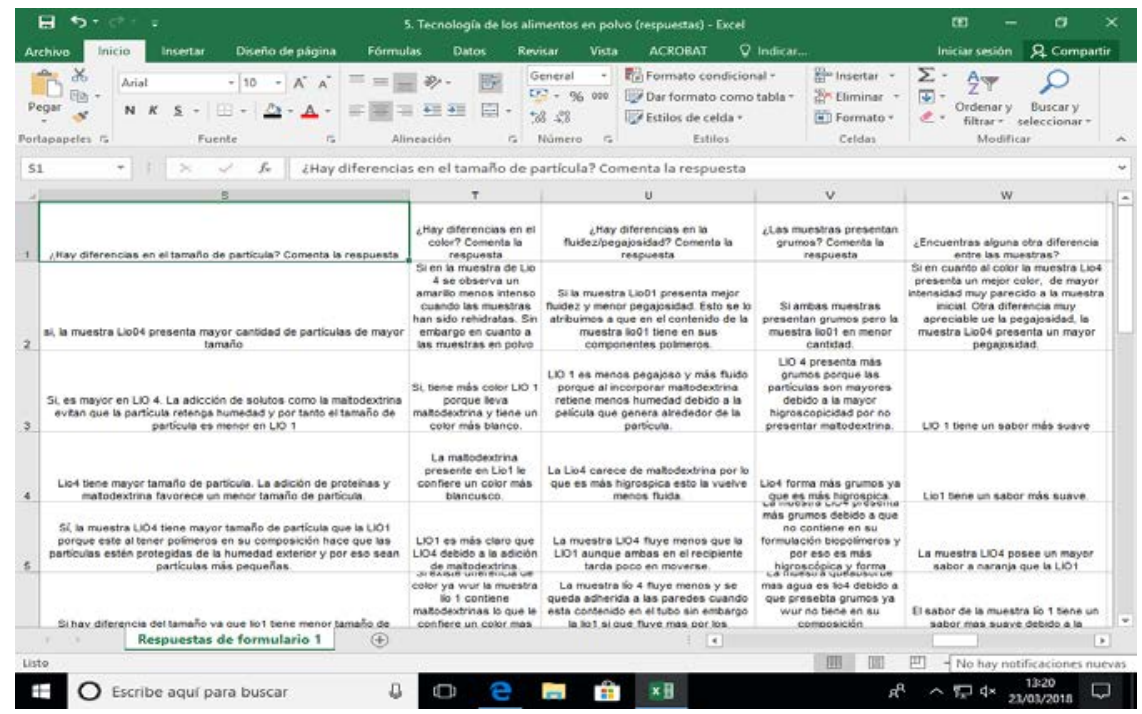

Fig. 10. Documento generado de la puesta en común de los resultados de las prácticas de laboratorio en el curso 2017-18.

Finalmente, los resultados obtenidos de la encuesta de satisfacción con la nueva metodología por parte de los alumnos muestran que el $75 \%$ de los alumnos valoraron positivamente el uso del móvil como herramienta para la ayuda a organizar la toma de datos en las prácticas de laboratorio y como facilitador del aprendizaje de la materia, mientras

(cc)) EY-NC-ND 2018, Universitat Politècnica de València

Congreso IN-RED (2018) 
que un $25 \%$ lo valoran negativamente. Por otra parte, parece que la utilización del móvil como guía les parece adecuada siendo valorado positivamente por el 81.3\% de los alumnos. Otro resultado interesante es que el $68.8 \%$ piensa que el uso del móvil ha aumentado su interés en la materia. Sin embargo, cuando se les pregunta si creen que el uso del móvil es más eficaz que el uso del sistema "tradicional" (lápiz y papel), el 31.3\% contestan que sí, el $31.3 \%$ que no y hay un $37.4 \%$ que contestan que tal vez. Esto puede ser debido a los problemas que encontraron y que quedaron plasmados en las sugerencias de mejora. En concreto echaron en falta la posibilidad de guardar la información, para evitar la pérdida de datos cuando se cerraba el programa (algo que ocurrió en algunos casos), y el consumo de batería del móvil. Por otra parte, comentaron que la utilización del móvil les había parecido interesante y útil pero que también era necesaria la toma de datos en papel y el uso de protocolos en las prácticas.

\section{Conclusiones}

La metodología docente debe adaptarse a la tecnología disponible en cada momento. En este sentido, hoy en día las tecnologías móviles están cambiando el panorama educativo, aportando movilidad, conectividad y permanencia. Estas interesantes características pueden favorecer, en un momento dado, al aprendizaje del alumno, ya que la generación de conocimiento depende de una adecuada gestión de la información y estos dispositivos ayudan a ello. Se trata, en cierta medida, de permitirles contar con una suerte de "aliado" que les ayude en la realización de las tareas que prescribe el profesor para la realización de la práctica. Algunos de los factores positivos que se derivaron del uso de los dispositivos móviles en el aula, como consecuencia de su implementación en el desarrollo de las prácticas de laboratorio, fueron el registro ordenado de los datos y la accesibilidad a la información en todo momento. Además, para el profesor, supuso una ventaja, a la hora de comprobar la asistencia y evaluar las prácticas. Por otra parte, se concluye que no se debe dejar de lado por completo la metodología “tradicional”, además de que es necesario mantener actualizada cualquier tecnología utilizada, para hacerla sostenible en el tiempo y cómoda en el manejo diario.

\section{Referencias}

ANDRES-FERRER, J. ALFARO-CID, E. LILLO, S. PEREZ DE LOS COBOS CASSINELLO, M. RODRIGO, A. (2013). El uso de encuestas rápidas a través del móvil en la enseñanza universitaria. Actas del XXI Congreso Universitario de Innovación Educativa en las Enseñanzas Técnicas. 1082-1092.

BAIN, K. (2007). Lo que hacen los mejores profesores universitarios. Valencia, Universitat de València.

GISBERT, M., ESTEVE, F. (2016). Digital Leaners: la competencia digital de los estudiantes universitarios. La Cuestión Universitaria, 7, 48-59.

2018, Universitat Politècnica de València

Congreso In-Red (2018) 
MORALES VALLEJO, P. (2008). Nuevos roles de profesores y alumnos, nuevas formas de enseñar y aprender. En, L. Prieto Navarro (coordinadora), La enseñanza universitaria centrada en el aprendizaje. Estrategias útiles para el profesorado. Barcelona, Octaedro-ICE.

MUÑOZ, M.J., BLASCO, E., BES, M.A., GARCÍA, J., SANCHIS, P., VIDAL, B., FENOLLOSA, M.L., RIBAL, F.J., TORTAJADA, L.A., NOGUERA, P. (2012). "Evaluación de las prácticas mediante rúbricas: experiencia multidisciplinar". Proceedings. Jornada de Innovación ICE-UPV. Valencia: Editorial UPV.

NOGUERA, P, TORTAJADA, L.A. ATIENZA, J, HERREERO, M.A. (2011). Autoevaluación previa a las prácticas de laboratorio químico: introducción al autoaprendizaje” en ARBOR Ciencia, Pensamiento y Cultura .Vol. 187 - Extra 3 diciembre. 267-272. 